\title{
Evolutionary Theory and the Epistemology of Science
}

\author{
Kevin McCain and Brad Weslake
}

\section{Introduction}

Evolutionary theory is a paradigmatic example of a well-supported scientific theory. In this chapter we consider a number of objections to evolutionary theory, and show how responding to these objections reveals aspects of the way in which scientific theories are supported by evidence. Teaching these objections can therefore serve two pedagogical aims: students can learn the right way to respond to some popular arguments against evolutionary theory, and they can learn some basic features of the structure of scientific theories and evidence.

We begin, in Sect. 2, with some general remarks about epistemology (the theory of knowledge) in order to help frame our discussion. After these brief remarks, in Sect. 3 we turn to the objections to evolutionary theory. In Sect. 4 we describe the epistemological lessons of these objections, and in Sect. 5 we conclude.

The authors contributed equally to this chapter

K. McCain ( $\triangle)$

Department of Philosophy, University of Alabama, 900 13th Street South,

Birmingham, AL 35294-1260, USA

e-mail: mccain@uab.edu

B. Weslake

Department of Philosophy, University of Rochester, Rochester, NY, USA

e-mail: bradley.weslake@ rochester.edu 


\section{Epistemological Background}

\subsection{The Traditional Account of Knowledge}

It is widely accepted by philosophers that in order to know some proposition, it must be true and you must believe it. However, to have knowledge one must satisfy more than these two conditions. To see this, consider the following case:

Sally makes a wild guess that there is now an even number of stars in the Milky Way galaxy and forms the belief that there is an even number of stars in the Milky Way galaxy on the basis of her guess.

Assume for the sake of illustration that Sally's guess happens to be true. Does Sally know that there is an even number of stars in the Milky Way galaxy? Surely not. After all, Sally has no reason to believe as she does. She is simply guessing, and she is aware that she has no good reasons for her belief. Not only does Sally fail to know that there is an even number of stars in the Milky Way galaxy, she is not even reasonable in believing that there is. Given her lack of evidence, the rational thing for Sally to do is to refrain from believing that there is an even number of stars and refrain from believing that there is not an even number of stars-in other words, she should suspend judgment concerning the number of stars. Since Sally has a true belief in this case, but fails to have knowledge, something more must be needed for knowledge in addition to true belief.

The fact that knowledge is not simply true belief has been recognized by philosophers since Plato. But what else is needed? In the Meno, Plato claims that what must be added to true belief is something that tethers one's belief to the truth. Many philosophers understand this "tether" to be the idea that one must have evidence that supports one's belief in order to have knowledge. ${ }^{1}$ Thus we arrive at what is sometimes called the traditional account of knowledge: one has knowledge of some proposition when the proposition is true and one believes the proposition on the basis of sufficiently strong evidence. ${ }^{2}$

\subsection{Evidence and Knowledge}

Of course, the traditional account of knowledge gives rise to an important question: how much evidence is required for knowledge?

\footnotetext{
${ }^{1}$ Some philosophers, e.g. Goldman (1999), would object to the idea that all knowledge requires evidence. However, most philosophers, Goldman included, accept that in order to have knowledge one's belief must be justified in some sense. Whatever that sense is, it must be capable of capturing everything we say below about the relationship between evidence and knowledge.

${ }^{2}$ See Feldman (2003) for further elaboration of the traditional account of knowledge as well as complications, such as the Gettier problem, that we are setting aside.
} 
One answer to this question is that one must have infallible evidence for a proposition in order to know that the proposition is true. Infallible evidence for a proposition is evidence that guarantees that the proposition is true. In other words, given infallible evidence, the proposition couldn't possibly be false. Although it is tempting to think that knowledge requires infallible evidence, this cannot be correct. At least, it cannot be correct if we have knowledge of much of anything. For the requirement of infallible evidence for knowledge leads to a thoroughgoing skepticism. We all accept that our perceptual experiences can fail to be accurate. That is to say, sometimes we are subject to illusions or hallucinations. For example, in the famous Müller-Lyer illusion, lines of the same length appear to be different lengths. Illusions such as this demonstrate that our perceptual experiences do not provide us with infallible evidence. Moreover, it is very plausible that, for any given perceptual experience, it might be misleading. So, for example, if you must have infallible evidence in order to have knowledge, you do not know that you are reading this book. After all, it is possible that you have the same evidence that you do (perceptual experiences) and yet are not reading this book. Thus, if infallible evidence is required for knowledge, we lack perceptually grounded knowledge of the world around us. ${ }^{3}$ This extreme skepticism is highly implausible. Thus, infallible evidence cannot be required for knowledge. Instead, some less demanding, fallible standard of evidence must be required for knowledge.

This is progress, but we are still left with a question: How much fallible evidence is required for knowledge? This is a difficult question that is still debated by epistemologists. Fortunately, for our purposes it is not necessary to give a precise answer. It is enough that we recognize that some standard of fallible evidence, rather than infallible evidence, is required for knowledge. That said, we note that a plausible rough characterization of the strength of fallible evidence required for knowledge is the "criminal standard" of evidence. ${ }^{4}$ According to this standard, in order to know a proposition is true one must have evidence that makes the truth of the proposition beyond a reasonable doubt. So, according to the criminal standard, knowledge requires evidence that is much weaker than infallible evidence, yet stronger than merely a good reason to believe. Although we grant that 'beyond a reasonable doubt' is somewhat vague, we will adopt this standard as a working guide for the degree of evidence required for knowledge.

At this point there is one final question about evidence and knowledge that it is worth briefly considering: What is it to be good evidence? There are many different kinds of evidence, so it would be a monumental task to try and say exactly what it is in virtue of which evidence is good evidence. In light of this, we will here only offer some brief remarks about good evidence. There are a variety of sources that provide good evidence. They include: logical and mathematical proofs, the sensory and introspective experiences of normally functioning humans, the testimony of

\footnotetext{
${ }^{3}$ For more on the nature of perceptual knowledge and the sort of evidence it requires see Alston (1993), Chisholm (1957), and Huemer (2001).

${ }^{4}$ This standard for the evidence required for knowledge is suggested by Conee and Feldman (2004).
} 
trustworthy sources, and controlled experiments. As is all too familiar, one's overall evidence can become better or worse with respect to a particular proposition. An obvious example is learning that one's evidence is defective. For example, when one has evidence from testimony and subsequently learns that the testifier is biased, this information can weaken or cancel the evidence from testimony. Similarly, learning that the results of an experiment are yielded by biased sampling can weaken or cancel the evidence provided by the experiment. On the other hand, ensuring that samples are fair and that testifiers are not biased can improve the strength of evidence that those sources provide. Finally, an important feature of evidential support is that the evidence for a proposition is much stronger when, other things being equal, the individual pieces of evidence come from independent sources. For example, when two consumer reports claim that a certain car is best, the evidence you gain is stronger when unrelated agencies publish the reports than it is when the same agency publishes both reports. These remarks are far from exhaustive, but they will suffice for our purposes. ${ }^{5}$

\section{Objections to Evolutionary Theory}

In this section we develop a characterization of the nature of science by responding to a set of objections that have been raised against evolutionary theory. We emphasize that these objections are not taken seriously by any scientists or philosophers of science. Moreover, they have recently been superseded in the public imagination by the arguments of intelligent design advocates (see Brigandt, this volume). We have chosen these objections not because they are plausible but because they are instructive. As we will argue in the remainder of this section, each misses something important about the nature of science.

Before we begin, we should clarify what we mean by evolutionary theory. We will say more about the structure of evolutionary theory overall below, but for now we can understand the theory to be composed of the following two hypotheses:

\footnotetext{
${ }^{5}$ It is worth noting that although we have been discussing what is required for knowledge, similar considerations apply to rational acceptability. Any time one lacks sufficient evidence for a proposition it is not rational for her to believe the proposition. Some may doubt this claim because they distinguish between things that someone should have beliefs about and things that someone should believe in. That is to say, they think that some propositions (the kind we have beliefs about) require evidence in support of them before they are rationally acceptable and some propositions (the kind we have beliefs in) do not require evidence for acceptability. Perhaps there is such a distinction between kinds of propositions and the evidential requirements for rationally accepting them (we doubt it). However, even if there are propositions that one should believe in without the need for supporting evidence, scientific theories are not among them. Scientific theories are accepted or rejected on the basis of evidence. If the evidence does not support a given scientific theory, then the theory should be rejected. So, when we discuss the rational acceptability of evolutionary theory we are assuming that rational acceptability requires having good evidence in support of the theory.
} 
1. Common Descent. All living organisms descended from a single common ancestor.

2. Natural Selection. Natural selection has been an important cause of the history of life.

The second hypothesis is vague, but is adequate for our purposes.

\subsection{Evolution Is a Mere Theory}

One criticism that is often leveled against evolutionary theory is that it is merely a theory, not a scientific fact. But what does this claim mean, and why should we believe it?

One defense of this claim is that evolutionary theory has not been "proved". ${ }^{6}$ The thought seems to be that a scientific fact is the sort of thing that has been proved true, whereas a theory is something that has not been proved true. ${ }^{7}$ Of course, defending the claim that evolutionary theory is a mere theory on the grounds that it has not been proved true raises an important question of its own-what is required for something to be proved? Clearly, it cannot be the case that in order for something to be accepted as a scientific fact we must possess a proof of the sort that can be found in logic or mathematics. This sort of proof involves showing that a conclusion follows deductively (it cannot possibly be false if the premises are true) from axioms that are self-evidently true. Proofs of this sort, if they exist at all, can only exist for truths of reason, which can be known without making observations of the universe around us. As we will show below, science does involve some claims that we can know in this way. But this cannot be all that science involves, because if it were, we would only be in a position to endorse very few scientific facts. Much of science makes claims about the universe around us as it happens to be. These claims are neither self-evidently true nor do they follow deductively from anything selfevidently true; instead they must be supported by observational evidence. So, it cannot be that proof of the logical or mathematical sort is required for something to be more than a mere theory.

Let us consider another way to make the distinction between a mere theory and a scientific fact. Perhaps the idea is that scientific facts are things that we know to be true, while mere theories are not known to be true. Now we distinguished above between three necessary conditions for knowledge (the proposition must be true, one must believe the proposition, and one's believing the proposition must be based on sufficient evidence). A proponent of this objection therefore must argue that at least one of the three conditions for knowledge is not met in the case of evolutionary theory. However, only the third condition is a plausible candidate for attack.

\footnotetext{
${ }^{6}$ See Watson (1976).

${ }^{7}$ Critics of evolution have sometimes been aided here by the careless statements of biologists, who have claimed that evolution has been demonstrated, or is indubitable (Kitcher 1982, p. 31).
} 
It is simply false or irrelevant to claim that evolutionary theory is not believed to be true. On one hand, it is straightforwardly false that supporters of evolutionary theory do not believe it. On the other hand, while it is true that some who object that evolutionary theory is only a theory do not believe it, this fact is irrelevant. For evolutionary theory to be known requires merely that someone believe it, while satisfying the other conditions for knowledge. It does not matter that critics of evolutionary theory fail to believe it.

Arguing that evolutionary theory is not true would be an effective way for objectors to show that it is merely a theory, on the interpretation of "merely a theory" under discussion. Unfortunately, there is no shortcut to the truth. The only way to argue that evolutionary theory is not true is to show that the evidence does not support it.

So the real question concerning whether evolutionary theory is only a theory is about evidence. Objectors can attempt to argue that evolutionary theory is only a theory because we do not have sufficient evidence to know that it is true.

We argued above that we should be fallibilists about the evidence required for knowledge. As we noted, this leaves us with difficult questions concerning exactly what strength of evidence is required for knowledge. Rather than trying to first settle this issue before determining whether evolutionary theory is only a theory, it is more practical to simply look at the evidence there is in support of evolutionary theory and see how that evidence compares to the evidence we have in support of other things that we know.

The first point to be made here is simply that there is an enormous amount of evidence in support of evolutionary theory. ${ }^{8}$ Moreover, the evidence comes from a very diverse array of independent sources. Regarding Common Ancestry, there is the evidence from the geographic distribution of species, the fossil record, the molecular record, embryology, comparative anatomy, and so on. ${ }^{9}$ Regarding Natural Selection, there is the evidence from experimental tests of evolution under natural selection in natural and laboratory contexts, together with work on mathematical models and computer simulations. ${ }^{10}$

To describe all this evidence and explain how it bears on the hypotheses that compose evolutionary theory is obviously far beyond the scope of this chapter. ${ }^{11}$ But it is helpful to consider two contrasts that may be made. One contrast is with knowledge claims made in ordinary life, and another contrast is with knowledge claims made elsewhere in science.

\footnotetext{
${ }^{8}$ Sometimes those who claim that evolution is only a theory seem to mean that evolution is a mere unsupported speculation, so we note in passing that the existence of this evidence is sufficient to show that the objection in this form is false. We thank Elliott Sober for reminding us of this interpretation.

${ }^{9}$ Good popular surveys of this evidence can be found in Coyne (2009) and Dawkins (2009). Carroll (2006) gives a popular survey of the evidence from the molecular record.

${ }^{10}$ For recent surveys of selection experiments, see Garland and Rose (2009) and Kawecki et al. (2012). For a survey of work involving computer simulations, see Adami (2006). There are myriad introductions to mathematical models of evolution, but for a concise survey of population genetics see Gillespie (2004).

${ }^{11}$ For an excellent treatment of the structure of the evidence, see Sober (2008).
} 
Compare the evidence in support of evolutionary theory to the evidence that we have for propositions we normally take ourselves to know. For example, we typically think that we can know things via the testimony of others either directly or through reading things that others have written. Consider the following sort of situation:

You want to see a particular movie at the local cinema. You check the showtimes in a local newspaper and it says that the movie starts at $7 \mathrm{pm}$.

Most people accept that in this situation you may come to know that the movie starts at $7 \mathrm{pm}$ by reading the newspaper. How does the evidence that you have in this situation stack up to the evidence in support of evolutionary theory? Intuitively, the evidence in support of evolutionary theory is much better (both in terms of quantity and strength) than the evidence you have for the showtime for the movie. After all, you only consulted a single newspaper and you do not have evidence about the typical showtimes of the local cinema. Further, it is not uncommon for local newspapers to occasionally have printing errors, nor is it uncommon for cinemas to change their showtimes. Despite the chance of error, it is plausible that you can know the showtime in this sort of situation. So it is also plausible that the strength of evidence in support of evolutionary theory is sufficient for knowledge.

Now compare the evidence for evolutionary theory with the evidence that warrants belief in other scientific theories. We can be said to know many scientific theories, but we will take as an example the discovery of electrons. ${ }^{12}$ This example provides a nice analogy with evolutionary theory. Like evolutionary theory, electron theory can be considered the conjunction of a number of independent hypotheses, e.g. that electrons are negatively charged, that they are much less massive than atoms, that they are of a single type, that they are constituents of all atoms. And like evolutionary theory, the type of evidence that elevated electron theory to the status of knowledge consisted in strong independent evidence for these core hypotheses. In the case of electron theory, the independent evidence included the deflection expected for negative charge in both electric and magnetic fields, the independent determination in these two fields of the ratio of mass to charge, and the robustness of these results across a wide range of experimental procedures and different gases. After J. J. Thomson presented these results in 1897, it was no longer reasonable to doubt the existence of electrons.

Now just as with evolutionary theory, there have since been radical changes in other aspects of the electron theory together with an impressive stability of these core hypotheses. The electron, like evolution, is now very far beyond a reasonable doubt. Our point is that if we compare the degree of evidence for electron theory at the time of Thomson with the degree of evidence for evolutionary theory now, it is extremely implausible that electron theory met, but evolutionary theory fails to meet, the evidential standards for knowledge.

\footnotetext{
${ }^{12}$ Our presentation of the discovery of electrons follows the lucid discussion of Norton (2000) and Bain and Norton (2001), who also consider the example in light of philosophical disputes concerning the very possibility of scientific knowledge.
} 
These considerations show that it is plausible that if we have the sort of knowledge that we typically take ourselves to have, both in everyday life and in science, we can have knowledge of evolutionary theory too. So one cannot plausibly claim that evolutionary theory is only a theory on the grounds that the evidence in support of evolutionary theory is insufficient. The evidence in support of evolutionary theory is comparatively stronger than the evidence we have in support of things that it is uncontroversial that we know. Thus, this objection to evolutionary theory rests on a misunderstanding of the degree of evidence required for something to be known.

There is a final point to be made about the objection that evolution is merely a theory. This phrase is often used by objectors to evolutionary theory as if there were some simple, easily determined property of evolutionary theory that makes it fit to be described as a mere theory. But even if it were true-contrary to the argument we have presented - that evolutionary theory failed to live up to the evidential standards for knowledge, there is no shortcut to this result. To argue that evolutionary theory fails to meet the evidential standards for knowledge would require detailed criticism of the entire range of evidence for the theory. This detailed criticism has yet to materialize.

\subsection{Evolution Is not Falsifiable}

A second criticism that has been leveled at evolutionary theory is that it is not falsifiable. ${ }^{13}$ This criticism traces back to an influential conception of the distinction between scientific and non-scientific theories due to Karl Popper (1963). According to this conception, a theory is scientific if and only if it is falsifiable. A theory is falsifiable, in turn, if and only if an observational consequence can be derived from the theory. The idea is that if such a consequence can be derived, then an observation designed to determine whether the consequence obtains provides a genuine test of the theory, and if the test fails then the theory must be false.

This criticism of evolutionary theory is therefore supposed to establish that evolutionary theory is not a scientific theory at all. The argument is as follows:

1. A theory is scientific if and only if it is falsifiable.

2. Evolutionary theory is not falsifiable.

3. Therefore, evolutionary theory is not scientific.

Given the definition of falsifiability introduced above, the critic of evolutionary theory claims that since it is not possible to derive an observation with the potential to establish that evolutionary theory is false, evolutionary theory is not falsifiable. Thus, the objection goes, evolutionary theory is not scientific at all.

There are two straightforward responses to this criticism. A first response is to reject the first premise of the argument. According to this response, falsifiability does not distinguish between scientific and non-scientific theories. A second response is

\footnotetext{
${ }^{13}$ See Gish (1979) and Morris (1974).
} 
to reject the second premise of the argument. According to this response, evolutionary theory is falsifiable after all. The first response is the correct one to make, as can be seen by considering the second response.

It is sometimes said by biologists (often under the influence of Popper) that evolutionary theory is falsifiable. ${ }^{14}$ Legend has it that the biologist J.B.S Haldane nominated the discovery of a rabbit fossil in Precambrian strata as something that evolutionary theory predicts will not occur. So let us consider whether such a discovery would falsify evolutionary theory. Notice that such a prediction cannot be deduced from Common Descent and Natural Selection alone. These propositions have no direct bearing whatsoever on where rabbits will be found (or, for that matter, on whether rabbits even exist). Rather, it is only in conjunction with other, more specific hypotheses that evolutionary theory makes predictions about where rabbits will be found. Philosophers of science refer to those other hypotheses as auxiliary hypotheses. The auxiliary hypotheses in this case include those that specify the evolutionary relationships between species, and those that specify the time-periods over which evolution has occurred. Since it is only in conjunction with auxiliary hypotheses that evolutionary theory makes predictions, the failure of such a prediction would not entail that evolutionary theory is false. Rather, at best it would entail that one of the cluster of hypotheses from which the prediction was derived is false. This point does not depend on anything special about fossil rabbits—all predictions of evolutionary theory are made by clusters of hypotheses, not by Common Descent and Natural Selection alone. In sum, evolutionary theory is not falsifiable, since it does not in isolation entail any predictions whatsoever.

It is important to be clear about this point. We have suggested that evolutionary theory is not falsifiable, in Popper's sense. This does not entail that evolutionary theory cannot be tested, but rather that falsifiability is an inadequate theory of testability (we discuss the idea that scientific theories should be testable in the following section). It also does not entail that the choice of which hypothesis to abandon in the face of a failed prediction is arbitrary. ${ }^{15}$ Our point is simply that no scientific theories are falsifiable in Popper's sense, and that this is a problem for Popper, not for our theories.

This leads us back to the first response. Does the unfalsifiability of evolutionary theory entail that it is not scientific? It is widely accepted by philosophers of science that it does not. For the feature of evolutionary theory in virtue of which it is not falsifiable is not unique, but rather is a feature of scientific theories in general. Hypotheses do not confront the world alone, but in groups. ${ }^{16}$ This point was clearly made in response to Popper by both Lakatos (1970) and Putnam (1974), who pointed out that Newton's theory of universal gravitation is unfalsifiable for exactly this reason. Newton's theory of universal gravitation alone implies nothing at all

\footnotetext{
${ }^{14}$ For a survey of Popper's influence on biologists, see Hull (1999).

${ }^{15}$ The problem of how to distribute belief in a cluster of hypotheses that are incompatible with an observation is known as the Quine-Duhem problem.

${ }^{16}$ For the classic presentation of this conception of the relationship between theories and predictions, see Duhem ([1914] 1954).
} 
about the motions of bodies, for it says nothing about where bodies are located, and about what other forces are acting. So it is not falsifiable. Moreover, as Lakatos and Putnam point out, when predictions made by Newton's theory in conjunction with accepted auxiliary hypotheses of these sorts failed, scientists frequently responded by rejecting the auxiliary hypotheses rather than the theory-and they were reasonable to do so. Likewise, it is possible that if a fossil rabbit were discovered in the Precambrian, it would be some of the auxiliary hypotheses to evolutionary theory that it would be reasonable to reject. This impugns the scientific status of neither Newton's theory nor Darwin's. It is false that a theory is scientific if and only if it is falsifiable. ${ }^{17}$

\subsection{Evolution Makes no Predictions}

We have argued that falsifiability is a poor criterion for judging whether a theory is scientific. However, our discussion also suggests a much better criterion for scientific theories, one that captures what might lead one to erroneously latch onto falsifiability. This criterion is that scientific theories must be testable. In order to be testable a theory must (in conjunction with appropriate auxiliary hypotheses, a qualification we will hereafter leave implicit) make predictions that can either be borne out or not. So, for a theory to be scientific, it must make predictions. This is another point on which evolutionary theory has been criticized. It has been claimed that evolutionary theory is not scientific because it does not make predictions. ${ }^{18}$

Although this criticism does at least challenge evolutionary theory from the standpoint of an accurate conception of scientific theories, it is hopelessly mistaken. Not only is evolutionary theory testable in virtue of the predictions it makes, it is one of the best tested theories in the history of science. While even a cursory survey is far beyond the scope of this chapter, we will mention two nice examples for the sake of illustration. First, after inspecting an orchid species from Madagascar with a foot-long nectary, Darwin predicted the existence of a moth with an extraordinarily long tongue, on the basis that orchids and moths had evolved together: "in Madagascar there must be moths with probosces capable of extension to a length of between 10 and 11 in.!" (Darwin 1862, p. 198). Even though Darwin remarked, in a later edition of the same work, that he had been ridiculed for this prediction by some entomologists, his prediction was finally confirmed in $1903 .{ }^{19}$ Of course, this illustrates a prediction the failure of which would hardly have been damaging to the core hypotheses of evolutionary theory. Second, there is a prediction that also

\footnotetext{
${ }^{17}$ Popper was aware of these objections, and revised his account of falsifiability in response. We will not discuss Popper's response, though we note that interpretations of falsifiability that increase the plausibility of the first premise of the argument under discussion also decrease the plausibility of the second premise. For other objections to Popper see Sober (2008, pp. 129-131).

${ }^{18}$ See Gish (1979) and Morris (1974).

${ }^{19} \mathrm{~A}$ brief presentation of this story can be found in Kritsky (1991).
} 
provides a lovely illustration of the power of independent lines of evidence. When the molecular structure of the gene was discovered and it was appreciated that phylogenies (descriptions of the genealogical relationships between species) could be constructed on their basis, it was an open question whether the older phylogenies constructed on the basis of morphological evidence would agree with the new phylogenies constructed from molecular evidence. Here are Zuckerkandl and Pauling (1965, p. 102), writing before the evidence was in:

It will be determined to what extent the phylogenetic tree, as derived from molecular data in complete independence from the results of organismal biology, coincides with the phylogenetic tree constructed on the basis of organismal biology. If the two phylogenetic trees are mostly in agreement with respect to the topology of branching, the best available single proof of the reality of macro-evolution would be furnished. Indeed, only the theory of evolution, combined with the realization that events at any supramolecular level are consistent with molecular events, could reasonably account for such a congruence between lines of evidence obtained independently $[\ldots]$

Zuckerkandl and Pauling put things too strongly, for as we have suggested, the success of this prediction would certainly not prove that the theory is true. Nevertheless, failure of the prediction would have been as dramatic as the discovery of a Precambrian rabbit, in the sense that many well-confirmed hypotheses would have had to come under scrutiny (Godfrey-Smith 2003, pp. 73-74). As it happens, the phylogenies constructed from molecular data are congruent with the older phylogenies to a remarkable degree..$^{20,21}$

Sometimes critics of evolutionary theory have something more specific in mind when they claim that evolutionary theory makes no predictions. This is the so-called "tautology problem", according to which evolutionary theory is not testable because it is equivalent to the thesis that the fittest organisms survive (in the sense that they leave more offspring). ${ }^{22}$ It is then claimed that the fitness of an organism is defined in terms of survival (in the sense that it is identified with number of offspring). Putting the thesis and definition together, the theory therefore is said to amount to the claim that the organisms that leave more offspring leave more offspring. This claim is tautologous, that is, it is true in virtue of logical form alone (the logical form here is something like: if $\mathrm{A}$ is $\mathrm{b}$, then $\mathrm{A}$ is $\mathrm{b}$ ). Tautologous claims cannot be tested, since they are necessarily true. Hence, the tautology problem.

The first thing to notice about this objection is that evolutionary theory cannot be reduced to the proposition that the fittest organisms survive. As we have presented the theory, it involves the hypotheses of Common Descent and Natural Selection.

\footnotetext{
${ }^{20}$ For a survey see Patterson et al. (1993), who write of the morphologists: "none of their wellsupported phylogenies is overthrown by molecular data". Of course, there is a huge amount of dispute concerning the precise details of the relationships between species. But the basic structure of the tree of life is supported by a vast array of independent lines of evidence (Cracraft and Donoghue 2004). Indeed, intense disagreement on the branches coupled with broad agreement on the trunk provides a nice metaphor for the state of evolutionary theory itself.

${ }^{21}$ There are interesting epistemological issues concerning just what propositions are tested by this evidence, and why. For discussion of these issues see Sober (2008, Chapter 4).

${ }^{22}$ See Morris (1974) and Wilder-Smith (1981).
} 
Neither of these hypotheses is tautologous. If there is a problem here, it must concern Natural Selection. According to Natural Selection, natural selection has been an important cause of the history of life. If natural selection reduces to the proposition that those who leave more offspring leave more offspring, would Natural Selection be untestable? It would not. For natural selection to cause a change in a population, there must be fitness differences in that population. If fitness is identified with number of offspring, then this is equivalent to there being differences in number of offspring in the population. Whether there are such differences is a testable proposition, as is whether such differences have been an important cause of the history of life. So even if the claim that the fittest survive were untestable, it would not follow that the proposition we have called Natural Selection is untestable. That a theory contains a tautology does not entail that the theory as a whole is a tautology (Sober 2000a, p. 71).

As it happens, it is also a mistake to think that fitness is always defined in terms of actual number of offspring. In many contexts, fitness is instead defined in terms of expected number of offspring. A simple thought experiment exhibits the attractions of this alternative definition (Scriven 1959, p. 478). Suppose that two identical twins with identical behavioural dispositions happen to be sitting next to one another, and a tree falls on one of them. The other goes on to reproduce. Should we assign different fitnesses to them? If fitness is identified with actual number of offspring we must, but if fitness is identified with expected number of offspring we can assign them the same fitnesses, and chalk up the difference in offspring to chance. It is considerations of this sort that have led philosophers to propose the so-called propensity interpretation of fitness, according to which fitness is identified with the propensity to leave a certain number of offspring (Mills and Beatty 1979; Sober 2000b).

There is a final point to be made about the role of fitness in evolutionary theory. Much of the work of modern evolutionary theory involves the construction of mathematical models of evolutionary processes, which involve assigning fitnesses of various different types to various different entities (Orr 2009). These models can be used to discover various surprising results, for example that if the fitnesses of two types vary with time, the type that dominates over the long term can depend not only on mean fitness, but on variance in fitness. Results such as these are analogous to tautologies in being necessarily true, since they are produced by mathematical proof. So evolutionary theory does contain some truths of reason, which cannot be tested. What can be tested is whether these mathematical models apply to actual biological populations. That evolutionary theory makes use of mathematical truths in this way is obviously a virtue rather than a defect of the theory.

\subsection{Evolution Has Been Falsified}

The final charge against evolutionary theory we will consider is that it has been falsified. Amusingly, some critics of evolutionary theory have argued both that is 
unfalsifiable and that it has been falsified ${ }^{23}$ It cannot be that both are true: if a theory has been falsified then it was falsifiable. We argued above that evolutionary theory is not falsifiable, and these arguments also suffice to show that evolutionary theory has not-indeed, that it cannot have-been falsified. Perhaps what is intended is the weaker thesis that evolutionary theory has not been well tested. Again, we have argued that this is a mistake. Evolutionary theory has been very well tested, partly in virtue of making a host of successful predictions such as those we described above. There is, however, a different argument that might lie behind the idea that evolution has been falsified. The argument is that evolutionary theory has made some predictions that have failed, and therefore that it should not be believed. It is this argument we will consider in this section.

We made the point earlier that evolutionary theory only makes predictions in conjunction with auxiliary hypotheses, and that in this respect the theory is typical. We also noted that in the case of Newton's theory of universal gravitation, when predictions made by the theory in conjunction with appropriate auxiliary hypotheses failed, frequently the reasonable response was for scientists to abandon the auxiliary hypotheses rather than the theory. A classic example is provided by the discovery of Neptune. Newton's theory in conjunction with accepted auxiliary hypotheses about the planets failed to accurately predict the orbit of Uranus. Rather than rejecting Newton's theory, scientists revised the auxiliary hypotheses to include the postulation of an additional planet, Neptune, which observations later confirmed. This example is already sufficient to show that the fact that a theory has made failed predictions does not entail that the theory should not be believed.

It is time to reveal an idealisation that we have been making about the nature of evolutionary theory. We have been treating the theory as consisting simply in the conjunction of the hypotheses of Common Descent and Natural Selection, and as surrounded by a host of auxiliary hypotheses that enable the theory to be tested. This is a picture that is associated with Lakatos (1970), who called these the "hard core" and "protective belt" of what he called scientific research programmes. But this is an oversimplification. In fact, evolutionary theory at any point in time consists in an enormous number of hypotheses. These hypotheses are located on a continuum from the more fundamental to the less fundamental, and are supported to different degrees. Different biologists pick out different subsets of these hypotheses as specifying evolutionary theory, depending on their purposes. For example, Ayala (1985, p. 59) distinguishes between three subsets of hypotheses. Common Descent and Natural Selection are members of the first subset. The second subset contains hypotheses about the specific relationships between species. The third subset contains hypotheses about the processes responsible for evolutionary changes. According to Ayala, the first subset contains the most fundamental claims of evolutionary theory, while those in the third subset are more peripheral. On the other

\footnotetext{
${ }^{23}$ In particular see Gish (1979), Morris (1974) and Wysong (1976) for creationist attacks on evolutionary theory that claim both that evolutionary theory is not falsifiable and that it has been falsified. See Kitcher (1982) and Sober (2000a) for discussion of the fact that creationists have leveled these inconsistent objections.
} 
hand, in an influential textbook on evolutionary theory, Futuyma (2009, pp. 9-11) identifies 16 "fundamental principles of evolution" that include principles concerning the genetic basis of evolutionary change and the processes responsible for speciation (the origin of new species). There is no reason to privilege any particular subset of these hypotheses as providing the real essence of evolutionary theory (Hull 1988). Ayala and Futuyma, in highlighting different subsets of the hypotheses accepted by most biologists, are not in disagreement.

This is important to recognize, since there is a strategy frequently used by critics of evolutionary theory to make it seem as if biologists themselves accept that evolutionary theory has been falsified. The strategy is to find some hypothesis that has been rejected, to claim that this hypothesis formed an essential part of evolutionary theory, and then conclude that evolutionary theory has been rejected. An interesting example of this strategy, since it strikes at Common Descent itself, is provided by the response of some critics to the discovery of so-called lateral gene transfer in prokaryotes. Some biologists have taken the discovery of lateral gene transfer to cast doubt on the existence of a single common ancestor of all life on Earth, understood as a single cell containing ancestors of all genes present today. ${ }^{24}$ Instead, it has been proposed that the common ancestor of life was a community of cells (Woese 1998). Critics of evolutionary theory have in turn seized this proposal as a refutation of a core hypothesis of evolutionary theory, Common Ancestry (see Doolittle 2009). According to these critics, biologists themselves have admitted that evolutionary theory is false.

So they have, if we take the exact statement of Common Ancestry to entail a single common cellular ancestor, and if this particular formulation of Common Ancestry is essential to the nature of evolutionary theory. But there is no reason to treat evolutionary theory as having an essence of this sort. If it turns out that the evidence supports the proposal that the common ancestor of life was a community of cells, we can replace Common Ancestry with that very similar hypothesis while preserving the bulk of the other hypotheses that compose evolutionary theory. To describe this possibility as involving the falsification of evolutionary theory is absurd, for the same reason it would be absurd to describe the quantum theory of the electron as involving the falsification of the existence of electrons, on grounds that it involved the reformulation of various classical hypotheses.

More generally, once we appreciate the vast range of hypotheses that compose evolutionary theory at any point in time, we are in a position to appreciate how it can be that biologists reject evolutionary hypotheses of various sorts all the time, while the core of the theory exhibits the remarkable stability we described above. The refinement and replacement of hypotheses on good evidential grounds is a mark of healthy science, not a sign of a failed theory.

\footnotetext{
${ }^{24}$ See Doolittle (2000) for a popular survey, and for philosophical reflections a special issue of Biology \& Philosophy on "The Tree of Life" edited by M. A. O'Malley (Vol. 25, No. 4, September 2010).
} 


\section{The Evidence for Evolution}

We have explained why various objections to evolutionary theory are unfounded and have explored some of the epistemic principles at work in scientific inquiry brought to light by consideration of these objections. In this final section we will briefly describe a principle of scientific inference that can be used to characterize the way in which evolutionary theory is supported by the evidence. This principle is inference to the best explanation.

In simplest terms inference to the best explanation involves the idea that explaining phenomena, just like predicting phenomena, provides evidence in support of a hypothesis. More precisely, the idea is that given a set of candidate hypotheses for some phenomena, the hypothesis that best explains the phenomena is the likeliest to be true. Of course, like all evidence, this evidence can be weakened or cancelled by other evidence.

There are difficult questions to ask about this principle, concerning what makes for good explanations, how it fits with general theories of confirmation, and why there should be a connection between good explanation and truth. Regardless of the answers to these questions, it is indisputable that inference to the best explanation is in fact deployed across a wide range of scientific disciplines (Glymour 1984, p. 173). It is also at the heart of some of the most famous episodes in the history of science, for example Antoine Lavoisier's argument against the phlogiston theory in favor of the oxygen theory of combustion, Christiaan Huygens' argument in support of the wave theory of light, and, of course, Charles Darwin's argument for evolution. ${ }^{25}$ In addition to being widely used in the sciences, inference to the best explanation is employed in everyday life: by jurors hearing a trial, by a doctor forming a diagnosis on the basis of a patient's symptoms, by someone's determining what is wrong with her computer. Indeed, inference to the best explanation is so pervasive in our reasoning that some have argued that it is a basic belief forming method for humans. ${ }^{26}$

As stated above, the principle merely says that the best explanation is the likeliest to be true. This does not entail that the best explanation should always be believed, for the likeliest may still be very unlikely. Warranted belief in the best explanation therefore requires in addition that the best explanation be good enough to warrant belief. That is to say, in order for a hypothesis to be legitimately inferred as true because it is the best explanation, it needs to meet certain minimal standards. ${ }^{27}$ Of course, it is a difficult task to make these minimal standards explicit. As with the degree of evidence required for knowledge, it is more practical to work with examples.

This brief characterization of inference to the best explanation is sufficient for us to see how it warrants accepting evolutionary theory on the basis of the evidence. For not only does evolutionary theory provide the best explanation for the evidence, it has no serious rivals. As Theodosius Dobzhansky famously said "nothing in

\footnotetext{
${ }^{25}$ See Thagard (1978) for a description of these arguments.

${ }^{26}$ See Enoch and Schechter (2008).

${ }^{27}$ For more on this see Lipton (2004).
} 
biology makes sense except in the light of evolution." ${ }^{28}$ That is to say, there is no rival theory that can provide anywhere near as good an explanation of the evidence as evolutionary theory. ${ }^{29}$ The fact that evolutionary theory has no serious rivals makes it easy for evolutionary theory to be the best available explanation-it is the best by default. Of course as we noted above, being the best available explanation is not sufficient for being accepted as true. The best available explanation must also be good enough. Is evolutionary theory a good enough explanation?

It is not an exaggeration to say that evolutionary theory offers one of the best explanations in the history of science. The sheer breadth of phenomena explained by the theory is astounding. As Ernst Mayr (1970, p. 1) writes, evolutionary theory "is quite rightly called the greatest unifying theory in biology. The diversity of organisms, similarities between kinds of organisms, patterns of distribution and behavior, adaptation and interaction, all this was a bewildering chaos of facts until given meaning by the evolutionary theory." Similarly, Philip Kitcher (1982, p. 50) writes that "the questions that evolutionary theory has addressed are so numerous that any sample is bound to omit important types." The explanatory and predictive power of evolutionary theory is simply incredible. Evolutionary theory provides an extremely good explanation of an enormous range of evidence, and there are no serious rival explanations. By inference to the best explanation, it is a paradigmatic case of a scientific theory that it is rational to accept.

\section{Conclusions}

We have explored a variety of objections to evolutionary theory. In every case we have shown that the objection is either clearly false or involves a misunderstanding of the nature of evidential support and scientific knowledge. In the process, we illuminated some of the epistemic principles that are at the heart of scientific inference, and showed how they are employed to establish the rational acceptability of evolution. We conclude by summarizing our central points-all of which are important for biology education because they help students to understand why evolutionary theory is a legitimate scientific theory and to understand some general features of good scientific theories:

- Knowledge requires true belief appropriately supported by evidence.

- Knowledge does not require infallible evidence. A working standard for knowledge is that one's evidence in support of a proposition (or theory) must make it beyond a reasonable doubt.

- The evidence for a theory is much stronger when, other things being equal, the individual pieces of evidence come from independent sources.

\footnotetext{
${ }^{28}$ Dobzhansky (1973).

${ }^{29}$ So-called intelligent design theories do not constitute genuine rivals (Sober 2008, Chapter 2; Brigandt, this volume).
} 
- Rational acceptability of a scientific theory cannot require the sort of proof that one can give in logic or mathematics, as this would render most scientific theories rationally unacceptable.

- Rational acceptability of any particular scientific theory should not require a level of evidence that cannot be met by other scientific theories, nor should it be so stringent that it would lead to a thoroughgoing skepticism.

- Scientific theories are tested in conjunction with auxiliary hypotheses, so a failed prediction does not entail that a theory is false. Hence, falsifiability is a poor criterion for scientific theories.

- In order to be scientific a theory should, in conjunction with auxiliary hypotheses, make testable predictions.

- The fact that a theory contains some tautologies or mathematical truths does not render the theory untestable.

- In order to be rationally acceptable at least some of a scientific theory's predictions must have been borne out.

- The fact that a theory explains relevant phenomena better than its rivals provides evidence in support of the theory.

- The fact that a theory is the best available explanation is not enough to support accepting the theory. The theory must also be a good explanation in its own right.

Acknowledgements We would like to thank Matt Frise, Kostas Kampourakis, Lynnette Lounsbury, H. Allen Orr, and Elliott Sober for their helpful comments.

\section{References}

Adami, C. 2006. Digital genetics: Unravelling the genetic basis of evolution. Nature Reviews Genetics 7(2): 109-118.

Alston, W.P. 1993. The reliability of sense perception. Ithaca: Cornell University Press.

Ayala, F. 1985. The theory of evolution: Recent successes and challenges. In Evolution and creation, ed. Mc.Mullin. Ernan, 59-90. Notre Dame: University of Notre Dame Press.

Bain, J., and J.D. Norton. 2001. What should philosophers of science learn from the history of the electron? In Histories of the electron: The birth of microphysics, Dibner Institute Studies in the History of Science and Technology, ed. Jed Z. Buchwald and Andrew Warwick, 451-466. Cambridge, MA: MIT Press.

Carroll, S.B. 2006. The making of the fittest: DNA and the ultimate forensic record of evolution. New York: W. W. Norton.

Chisholm, R. 1957. Perceiving. Ithaca: Cornell University Press.

Conee, E., and R. Feldman. 2004. Making sense of skepticism. In Evidentialism, 277-306. New York: Oxford University Press.

Coyne, J.A. 2009. Why evolution is true. New York: Viking.

Cracraft, J., and M.J. Donoghue (eds.). 2004. Assembling the tree of life. Oxford: Oxford University Press.

Darwin, C. 1862. On the various contrivances by which British and foreign orchids are fertilised by insects. London: John Murray.

Dawkins, R. 2009. The greatest show on earth: The evidence for evolution. New York: Free Press.

Dobzhansky, T. 1973. Nothing in biology makes sense except in the light of evolution. The American Biology Teacher 35: 125-129. 
Doolittle, W.F. 2000. Uprooting the tree of life. Scientific American 282(6): 90-95.

Doolittle, W.F. 2009. The practice of classification and the theory of evolution, and what the demise of Charles Darwin's tree of life hypothesis means for both of them. Philosophical Transactions of the Royal Society B: Biological Sciences 364(1527): 2221-2228.

Duhem, P. 1954/1914. The aim and structure of physical theory. Princeton: Princeton University Press.

Enoch, D., and J. Schechter. 2008. How are basic belief-forming methods justified? Philosophy and Phenomenological Research 76(3): 547-579.

Feldman, R. 2003. Epistemology. Upper Saddle River: Prentice-Hall.

Futuyma, D.J. 2009. Evolution, 2nd ed. Sunderland: Sinauer Associates.

Garland Jr., T., and M.B. Rose. 2009. Experimental evolution: Concepts, methods, and applications of selection experiments. Berkeley: University of California Press.

Gillespie, J.H. 2004. Population genetics: A concise guide, 2nd ed. Baltimore: Johns Hopkins University Press.

Gish, D.T. 1979. Evolution? The fossils say no! San Diego: Creation-Life Publishers.

Glymour, C. 1984. Explanation and realism. In Scientific realism, ed. Jarrett Leplin, 173-192. Berkeley: University of California Press.

Godfrey-Smith, P. 2003. Theory and reality: An introduction to the philosophy of science, 2 nd ed. Chicago: University of Chicago Press.

Goldman, A. 1999. Internalism exposed. Journal of Philosophy 96(6): 271-293.

Huemer, M. 2001. Skepticism and the veil of perception. Lanham: Rowman \& Littlefield Publishers, Inc.

Hull, D.L. 1988. Science as a process: An evolutionary account of the social and conceptual development of science. Chicago: University of Chicago Press.

Hull, D.L. 1999. The use and abuse of Sir Karl Popper. Biology and Philosophy 14(4): 481-504.

Kawecki, T.J., et al. 2012. Experimental evolution. Trends in Ecology and Evolution 27(10): 547-560.

Kitcher, P. 1982. Abusing science: The case against creationism. Cambridge, MA: MIT Press.

Kritsky, G. 1991. Darwin's Madagascan Hawk Moth prediction. American Entomologist 37(4): 206-210.

Lakatos, I. 1970. Falsification and the methodology of scientific research programmes. In Criticism and the growth of knowledge, proceedings of the international colloquium in the philosophy of science, vol. 4, ed. Imre Lakatos and Alan Musgrave, 91-196. Cambridge: Cambridge University Press.

Lipton, P. 2004. Inference to the best explanation, 2nd ed. New York: Routledge.

Mayr, E. 1970. Populations, species, and evolution. Cambridge, MA: Harvard University Press.

Mills, S.K., and J.H. Beatty. 1979. The propensity interpretation of fitness. Philosophy of Science 46(2): 263-286.

Morris, H.M. 1974. Scientific creationism. San Diego: Creation-Life Publishers.

Norton, J.D. 2000. How we know about electrons. In After Popper, Kuhn and Feyerabend, Recent issues in theories of scientific method, Australasian studies in history and philosophy of science, vol. 15, ed. Robert Nola and Howard Sankey, 67-97. Dordrecht: Kluwer.

Orr, H.A. 2009. Fitness and its role in evolutionary genetics. Nature Reviews Genetics 10(8): 531-539.

Patterson, C., D.M. Williams, and C.J. Humphries. 1993. Congruence between molecular and morphological phylogenies. Annual Review of Ecology and Systematics 24(1): 153-188.

Popper, K. 1963. Conjectures and refutations. London: Routledge and Kegan Paul.

Putnam, H. 1974. The 'corroboration' of theories. In The philosophy of Karl Popper, Library of living philosophers, vol. 14, ed. Schilpp Paul Arthur, 221-240. La Salle: Open Court.

Scriven, M. 1959. Explanation and prediction in evolutionary theory: Satisfactory explanation of the past is possible even when prediction of the future is impossible. Science 130(3374): 477-482.

Sober, E. 2000a. Philosophy of biology, 2nd ed. Boulder: Westview Press. 
Sober, E. 2000b. The two faces of fitness. In Thinking about evolution: Historical, philosophical, and political perspectives, ed. Singh Rama Shankar et al., 309-321. Cambridge: Cambridge University Press.

Sober, E. 2008. Evidence and evolution: The logic behind the science. Cambridge: Cambridge University Press.

Thagard, P. 1978. The best explanation: Criteria for theory choice. Journal of Philosophy 75(2): 76-92.

Watson, D.C.C. 1976. The great Brain Robbery. Chicago: Moody Press.

Wilder-Smith, A.E. 1981. The natural sciences know nothing of evolution. San Diego: CreationLife Publishers.

Woese, C. 1998. The universal ancestor. Proceedings of the National Academy of Sciences 95(12): 6854-6859.

Wysong, R.L. 1976. The creation-evolution controversy. Midland: Inquiry Press.

Zuckerkandl, E., and L. Pauling. 1965. Evolutionary divergence and convergence in proteins. In Evolving genes and proteins, ed. Vernon Bryson and Henry J. Vogel, 97-166. New York: Academic. 